\title{
REVEALING THE PHYSICAL CHANGES TAKING PLACE IN Z-TRACK MOTION IN LOW MASS X-RAY BINARIES
}

\author{
M. Bałucińska-Church, ${ }^{1,2}$ M. J. Church, ${ }^{1,2}$ G. Halai, ${ }^{1}$ and A. Szostek ${ }^{3}$
}

The explanation of the strong physical changes clearly taking place in the Z-track class of Low Mass X-ray Binaries has so far not been obtained, and this remains a significant astrophysical problem, without which we cannot claim to understand accretion in I.MXB. We have for the first time applied the Birmingham emission model $(2,3)$ to this problem to attempt to obtain a solution from the spectral evolution along the $\mathrm{Z}$-track in the source GX 340+0 observed with Rossi$X T E$. In this model, $\mathrm{X}$-ray emission consists of blackbody from the neutron star, plus Comptonized emission from an extended ADC.

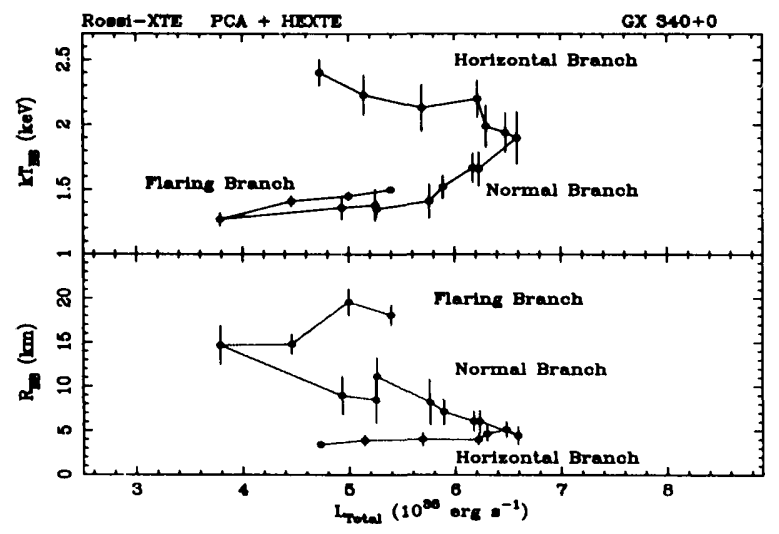

Fig. 1. Neutron star blackbody temperature and radius: evolution along the Z-track in GX $340+0$

The results immediately demonstrate that strong changes take place on the neutron star and inner disk during Z-track movement. Siarting from the one $\mathrm{x}$ treme end of the Z-track on the Horizontal Branch. the neutron star blackbody temperature $k T_{\mathrm{BB}}$ is initially high at the Eddington temperaure, and the emitting area very small, indicating that radiation pressure effects are strong as we have found in other sources (1). However, along the Normal Branch, the source clearly recovers from these effects with $k T_{\mathrm{BB}}$ falling and the blackbody radius increasing $R_{\mathrm{BB}}$ to

\footnotetext{
${ }^{1}$ School of Physics \& Astronomy, University of Birmingham, Birmingham B15 2TT. UK (mbc@star.sr.bham.ac.uk).

${ }^{2}$ Astronomcal Observatory, Jagiellonian University, ul Orla 171, 30-244 Cracow, Poland.

${ }^{3}$ Copernicus Astronomical Centre, ul. Bartycka 18, 00-716 Warsaw, Poland.
}

the full radius of the neutron star. In flaring, the radius increases further due to the release of energy on the surface of the neutron star.

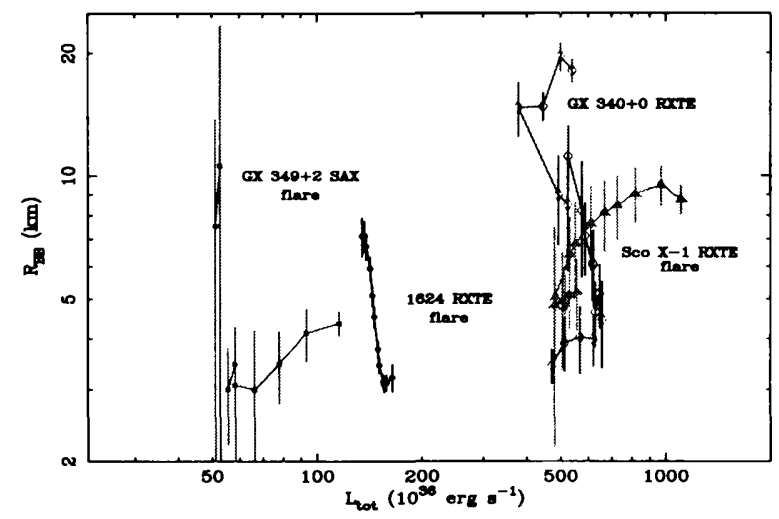

Fig. 2. Comparison of GX $340+0$ with several Z-sources and the dipping, flaring source X 1624-490

In Fig. 2, we compare these results for $R_{\mathrm{BB}}$ in GX $340+0$ with previous results for other Z-track sources and with the flaring in the dipping source X 1624-490, showing that flaring follows the same pattern as in Sco X-1, except that in Sco X-1, the maximum value of $R_{\mathrm{BB}}$ is $9.6 \mathrm{~km}$ consistent with the neutron star radius, whereas in GX $340+0$, the radius increases to more than $20 \mathrm{~km}$, as seen in a very small number of Galactic sources, implying that the emitter is a cloud of hot material enveloping the neutron star. The most interesting question is what drives the above recovery process. A clear possibility is that the mass accretion rate $\dot{M}$ to the neutron star decreases on the Normal Branch; however, this contrasts with the assumption widely made that $\dot{M}$ increases monotonically from the Horizontal to the Flaring Branch.

Acknowledgments: This work was supported in part by the Polish KBN grants PBZ-KBN054/P03/2001 and KBN-1528/P03/2003/25

\section{REFERENCES}

1. Bałucińska-Church, M., Barnard, R., Church, M. J. \& Smale, A. P. 2001, A\&A, 378, 847

2. Church, M. J. \& Batucińska-Church, .I. 2001, A\&A, 369,915

3. Church, M. J. \& Bałucińska-Church, M. 2004, MNRAS, 348,955 